\title{
Guideline
}

Sylvie Crauste-Manciet*, Irene Krämer, Frederic Lagarce, Valerie Sautou, Alison Beaney, Julian Smith, V'lain Fenton-May, Jean-Daniel Hecq, Farshid Sadeghipour and Paul Le Brun

\section{GERPAC Consensus Conference - Guidance on the Assignment of Microbiological Shelf-life for Hospital Pharmacy Aseptic Preparations}

https://doi.org/10.1515/pthp-2020-0001

Received February 07, 2020; accepted February 12, 2020

\begin{abstract}
All dosage forms prepared in hospital pharmacies should be labelled with an appropriate shelf-life. This shelflife should be validated taking chemical, physical and microbiological data into consideration. This guidance focuses on parenteral aseptically prepared products, as they are highrisk preparations. The risk is exacerbated by a requirement for longer shelf lives for reasons of economy and efficiency. The scope of this guidance includes individual patient preparations, preparations prepared in series (same type of
\end{abstract}

\footnotetext{
*Corresponding author: Sylvie Crauste-Manciet, ARNA U1212 INSERM UMR 5320, University of Bordeaux, 146 rue léo Saignat, Bordeaux 33076, France,

E-mail: sylvie.crauste-manciet@u-bordeaux.fr https://orcid.org/0000-0002-9817-2825

Irene Krämer, Department of Pharmacy, University Medical Center, Langenbeckstrasse 1, Mainz 55131, Germany,

E-mail: irene.kraemer@unimedizin-mainz.de

Frederic Lagarce, Department of Pharmacy, University Hospital of Angers, 4 rue Larrey, Angers 49033, France,

E-mail: frederic.lagarce@univ-angers.fr

Valerie Sautou, ICCF UMR 6296 CNRS, Faculty of pharmacy, Clermont Auvergne University, 28 Place Henri Dunant, Clermont-Ferrand 63000, France, E-mail: valerie.sautou@uca.fr Alison Beaney, Stockton QC Laboratory, University Hospital of North Tees., Harwick, Stockton, Stockton-on-Tees TS19 8PE, UK,

E-mail: alison.beaney@nhs.net

Julian Smith, JCS Pharma Consulting Ltd, Yew Tree Cottage, Hendrew Lane, Llandevaud, Newport, Gwent, NP18 2AB, UK,

E-mail: juliancsmith@outlook.com

V'Iain Fenton-May Former Quality Control Pharmacist to the Welsh Hospitals Cardiff United, Cardiff, UK, E-mail: vfentonmay@gmail.com

Jean-Daniel Hecq, Pharmacym, CHU UCL Namur, Avenue Therasse, Yvoir, Namur 5530, Belgium, E-mail: jean-daniel.hecq@skynet.be Farshid Sadeghipour, Department of Pharmacy, Lausanne University Hospital, Bugnon, 46, Lausanne, VD 1011, Switzerland,

E-mail: farshid.sadeghipour@chuv.ch

Paul Le Brun, Department of Clinical Pharmacy \& Toxicology, Leiden University Medical Center, Albibusdreef 2, Leiden 2300 RC, Netherlands, E-mail: p.p.h.le_brun@lumc.nl
}

preparation being repeatedly prepared) and batch preparations prepared from the same initial bulk admixture.

Keywords: shelf-life, microbiological, consensus, guidance

\section{Introduction and scope}

All dosage forms prepared in hospital pharmacies should be labelled with an appropriate shelf-life. This shelf-life should be validated taking chemical, physical and microbiological data into consideration.

This guidance focuses on parenteral aseptically prepared products, as they are high-risk preparations. The risk is exacerbated by a requirement for longer shelf lives for reasons of economy and efficiency. The scope of this guidance includes individual patient preparations, preparations prepared in series (same type of preparation being repeatedly prepared) and batch preparations prepared from the same initial bulk admixture.

\section{Background considerations}

In the monograph on Pharmaceutical Preparations (Ph. Eur. 9.0/2619), the European Pharmacopoeia (Ph. Eur.) states: "Health care professionals involved in unlicensed preparations have a duty of care to the patient receiving these preparations: a risk assessment is required to determine the extent and significance of testing."

Based on this risk assessment, the person responsible for the preparation must ensure that the pharmaceutical preparation is fit for purpose throughout its shelf life. Storage conditions and shelf lives must be justified based on physicochemical and microbiological stability. Published experimental data may be available to support the shelf life assigned to a preparation. In the absence of data, professional judgement is required. Numerous publications regarding the physicochemical stability of ready-to-use and ready-to- 
administer aseptic preparations prepared in hospital pharmacies are available in specific databases. Publications about the microbiological stability of these aseptic preparations are limited. Therefore, GERPAC (European Scientific Society specialised in Pharmaceutical Technology) decided to address this question with the help of a European expert working group.

\section{Microbiological quality of pharmaceutical preparations}

Sterile pharmaceutical preparations must be prepared with starting materials and conditions that ensure sterility of the preparations, in accordance with the Ph. Eur. The European Pharmacopoeia monograph, Test for sterility, generally requires that $10 \%$ of a batch is sampled for sterility testing. This is often impractical, as many aseptic preparations are prepared for individual patients. The Pharmacopoeia does not require all batches (see definitions Box 3) of pharmaceutical preparations to be tested for sterility but, if tested, every batch should comply. The microbiological sterility of an aseptically prepared product must be assured including robust container integrity data.

Detailed recommendations are given in PIC/S PE 010 'Guide to good practices for the preparation of medicinal products in healthcare establishments, Annex 1 Guidelines on the standards required for the sterile preparation of medicinal products'.

\section{Microbiological risk factors for different types of pharmaceutical preparations}

The risk of microbiological contamination for aseptic preparations is increased if the method of preparation is complex. Complex preparations involve more than five aseptic non-touch manipulations, or an open system, where the sterile medicine is exposed to the environment. The consequences of any microbiological contamination introduced during preparation are more severe if the preparation is susceptible to microbiological growth, and it is not used immediately. ${ }^{1}$

1 Cf. Closed system definition and complex calculation: Resolution CM/Res(2016)1.
Risk factors for the microbiological quality of pharmaceutical preparations include the nature of the product (see Box 1). For example, parenteral nutrition is susceptible to microbiological growth and is administered over several hours. This is considered to be a high risk preparation. By contrast, an antibiotic that involves a simple reconstitution process using closed transfer process and is given as a bolus dose is generally considered to be a low risk preparation.

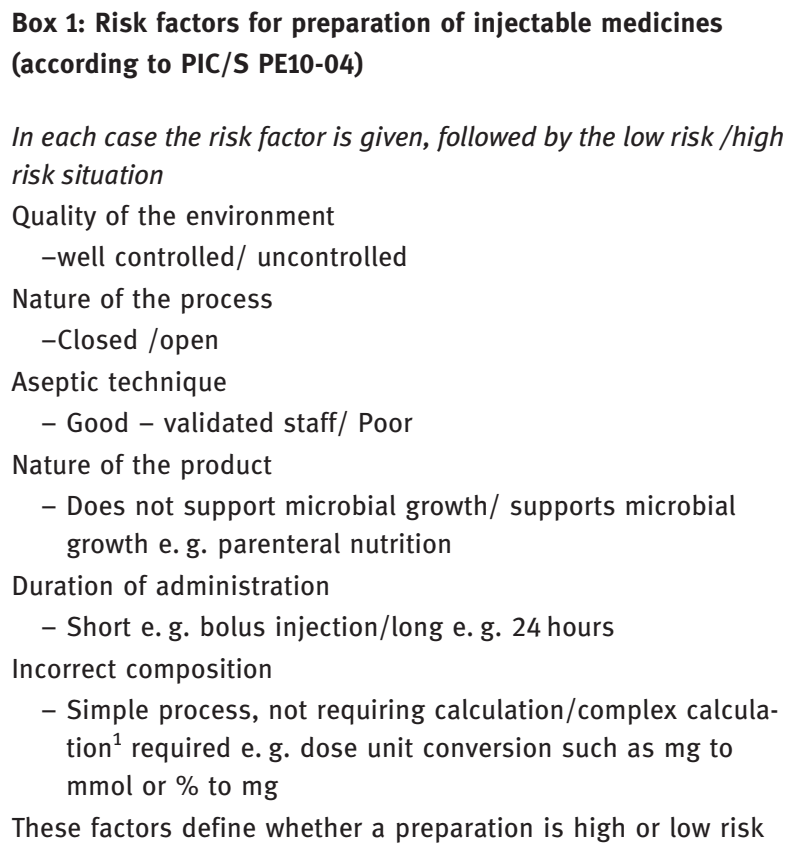

- Does not support microbial growth/ supports microbial growth e. g. parenteral nutrition

Duration of administration

- Short e. g. bolus injection/long e. g. 24 hours

Incorrect composition

- Simple process, not requiring calculation/complex calculation ${ }^{1}$ required e. $\mathrm{g}$. dose unit conversion such as $\mathrm{mg}$ to $\mathrm{mmol}$ or \% to $\mathrm{mg}$

These factors define whether a preparation is high or low risk

To maintain the sterility of the components and to ensure that the final preparation is sterile, careful attention needs to be given to the following factors: environment; premises; personnel; critical surfaces; sterility of container/closure, transfer procedures; disinfection procedures; the maximum holding period of the preparation before filling into the final container. Regular process validation is required to ensure these factors are considered and are under control. This validation will include process simulation tests using microbial growth media.

The use of microbial growth media for simulations refers to 3 different possible objectives (please refer to box 2.)

Box 2: A guide to Simulations with a Microbial Growth Medium Replacement of pharmaceutical starting materials with a microbiological growth medium, for example tryptone soya broth, for aseptic manipulation occurs in three different circumstances:

1. An entire process, at maximum batch size i. e. a 'worst case', can be simulated with a microbiological growth medium then incubated as part of process validation. The test should simulate the regular preparation in terms of equipment, processes, personnel involved, and filling period, as well as any holding 
times. The process simulation test should represent a "worst case" scenario and include all manipulations and interventions likely to occur during a preparation "session". This test is sometimes called a 'media fill'.

2. A shortened version of process validation i. e. simulation of some of the key manipulation steps in a process (but not at maximum batch size), followed by incubation, can be used at the end of an aseptic work session, as part of the on-going monitoring programme, to give assurance of sterility in place of sterility testing of a product sample. This may be known as an 'end of session broth test' or 'on-going simulation test'.

3. Manipulation of a microbiological growth medium is also used to regularly assess the aseptic technique of operators to ensure that the operator can maintain sterility during manipulations. This is known as an 'operator broth transfer validation test'. Initial competence of an operator should be established by the successful completion of at least three consecutive tests of this type, with regular re-assessment as part of an on-going programme.

Environmental risk factors and the results of ongoing environmental monitoring should be considered when assigning a shelf life to aseptic preparations. Other risk factors include the susceptibility of the preparation to microbial growth, storage conditions, container integrity, staff training, gowning technique, bioburden of materials and equipment, and the complexity of processing.

\section{Definition of a batch regarding microbiological testing}

Microbiological tests should be based on the definition of a batch (see Box 3).

For microbiological testing of parenteral pharmaceuticals prepared in hospital pharmacy, a batch may be defined as preparations prepared during the same session, under uniform process conditions. A session is defined as a period of time where the preparation process can be reasonably expected to present a uniform risk of contamination to the final preparation(s). Typically, a session is the period of continuous work between breaks and is not longer than a morning or an afternoon

2 Methodological guidelines for stability studies of hospital pharmaceutical preparations. GERPAC-SFPC First Ed October 2013, 71 pp ISBN: 978-2-9526010-4-7

Available on line at https://www.gerpac.net/platform/course/view. php?id=11 accessed December 2019.
Box 3: Batch definitions (from GERPAC guide) ${ }^{2}$

"in hospital pharmacy, the definition of a batch can vary considerably and is closely related to the type of preparation. The batch can either comprise a number of homogenous units of the same composition intended for the same or various patients or consist of a single unit intended for a single patient, prepared manually or by a (semi) automated method.

Two types of batches are:

The batch defined by the type of preparation

The batch comprises:

- Several final preparations of the same composition, filled from the same initial bulk admixture,

- A single final preparation for a single patient.

The batch defined by the mode of preparation

The batch comprises:

- several final preparations prepared under identical aseptic conditions and by the same staff.

\section{Microbiological stability studies of aseptic pharmaceutical preparations during preparation development}

Microbiological stability of aseptically-prepared pharmaceutical preparations is assessed by a combination of validation tests to be undertaken during preparation development. These validation tests, or process validation, are performed using broth culture media and by integrity testing of the final container. Integrity testing of the final container includes physicochemical and microbiological methods. When these validation tests are successfully completed, finished product tests on the final preparations must be performed, including sterility testing and endotoxin testing, if relevant.

Validation using the finished product itself, rather than by simulation, is mandatory when the preparation to be developed is known to affect the integrity of the container, for example by leaching.

Microbiological process validation is performed by Media Fill Test (MFT) using culture media instead of the preparation components. The microbiological quality of the environment is assessed concurrently to MFT. Operators involved in MFT should be previously validated by an operator broth transfer validation test. (See Box 2). The process simulation with a broth culture medium is important to demonstrate that the process results in preparations free from microbiological contamination under worst-case 
circumstances. This simulation testing may to be used during the integrity testing assessment of the container. The simulation must reproduce the whole procedure and its different steps.

The information provided here is only intended as a brief introduction to the use of broth for simulation testing and process validation of aseptic preparation. The Note for Guidance on process Validation ${ }^{3}$ should be read for further details. See Box 4 for summary on process validation with media fill

\footnotetext{
Box 4. Process validation using media fill

In aseptic preparation in hospital pharmacies, the number of containers filled during the process validation in general equates to the typical batch size of that product or process. The actual number of MFT depends on individual circumstances but generally consists of three batches per "process" and should be carried out before routine manufacturing or preparation can start.

The finished containers are incubated at $20-25^{\circ} \mathrm{C}$ for a minimum of 7 days followed immediately, or after a first reading, by incubation at $30-35{ }^{\circ} \mathrm{C}$ for a total minimum incubation time of 14 days. Other incubation schedules are acceptable if supported by scientific data (for example 2 weeks incubation time at $30^{\circ} \mathrm{C}$ is established in the Netherlands).

All containers must be free from microbial growth (indicated by turbidity) at the end of the incubation period.

Considering the small batch size usually prepared in hospital pharmacies, any positive result should result in the failure of the MFT and the source of contamination must be investigated, resolved and the MFT successfully repeated.
}

In aseptic manufacturing of licensed products, process validation takes place with a large number of units (at least 5000), to demonstrate that the risk of microbiological contamination is less than $0.1 \%$. With smaller batches it is permissible to use the actual batch size in the simulation. Since the number of units in a batch of aseptic preparations in a hospital environment is always smaller than 5000, an alternative "continuous process

3 Note for Guidance on Process Validation (EMA, 2001)

Guidance for Industry for the Submission Documentation for Sterilization Process Validation in Application in Applications for Human and Veterinary Drug Products (Technical Report Series No. 957, 2010)

Guidance for Industry; Sterile Drug Products Produced by Aseptic Processing - Current Good Manufacturing Practice (FDA, September 2004)

Recommendation on the Validation of Aseptic Process (PIC/S, January 2011)

Guide to Good Manufacturing Practice for Medicinal Products Annexes (PIC/S, September 2009)

EC Guide to Good Manufacturing Practice (Annex 1) March 2009. simulation" can be introduced to confirm the validity of the process. (see box 5).

\section{Box 5. Ongoing validation by end of session broth test}

The continuous end of session broth test has been developed for batches that consist of only a few units, including batches of one single unit.

The test consists of broth simulations of one or more aseptic manipulations. The manipulations should be chosen so that they are representative of all the usual aseptic actions.

The frequency that the test is carried out depends on the way in which the validation is performed. For example, one broth simulation process at the end of a working day, or several broth simulations at the end of a routine working session at defined intervals (e. g. once per week).

If there is no growth from 300 tests, this means that the chance of contamination is less than or equal to $1 \%$ ( $95 \%$ reliability). This is the minimum level that must be achieved. ${ }^{4}$

\section{Microbiological and physical integrity testing of the final container}

Integrity testing of the final container may be performed with broth medium both for physical and microbiological integrity tests. In case of identified risks of chemical interaction between the pharmaceutical substance and the final container, physical testing should be performed with the drug intended to be produced.

The integrity of the final container is essential to maintain sterility of a preparation. The purpose of integrity testing is to demonstrate that the container/closure system, such as the syringe and blind hub (cap), protects the preparation against ingress of microorganisms during storage and transport. Protocols are available for both microbiological and physical integrity testing methods. ${ }^{5}$

To ensure an adequate and realistic challenge, integrity testing methods must be active, rather than merely passive. Passive methods involve filling the container with nutrient broth and merely storing, and possibly transporting, them for the test period. The test fails if turbidity of the nutrient

4 Boom FA, Beaney AM. Aseptic handling. In: Bouwman-Boer Y, Fenton-May V, le Brun PPH eds. Practical pharmaceutics. Switzerland: Springer International Publishing, 2015: 695-706.

5 Protocols for the Integrity Testing of Syringes, NHS Pharmaceutical Quality Assurance Committee, Ed 2 April 2013 Available at: https://www.sps.nhs.uk/articles/microbiological-proto col-for-the-integrity-testing-of-syringes/). 
broth is observed, indicating microbiological growth. By contrast, active microbiological integrity testing methods (see box 6) involve immersion of the broth-filled final containers in a suspension of specific microorganisms. Escherichia coli (E.coli) is often used as the challenge organism because of its motility, which allows it to penetrate small gaps. More recently, Brevundimonas diminuta has been used as it is the smallest available test organism. The broth-filled final containers are immersed, either completely or partially, in the suspension of the chosen bacteria. If syringes are used as primary container, it is best to test both the syringe hub closed with a cap and plunger, so total immersion is preferable. Partial immersion may be useful in order to identify the site of ingress if total immersion fails the test.

BOX 6. Microbiological integrity testing example in practice for syringes

Prepare a suitable number of containers (e.g. a batch, or at least 20) in the aseptic unit to contain sterile Tryptone Soya Broth (TSB) in place of the preparation, noting the details of the syringe and blind hub/cap manufacturer and batch number. Pre-incubate these broth-filled containers at $20-25{ }^{\circ} \mathrm{C}$ for 7 days, then $30-35{ }^{\circ} \mathrm{C}$ for 7 days to ensure that the aseptic fill has been carried out correctly and the contents are sterile.

Discard any containers showing turbidity or microbial growth.

Prepare a pure culture of the chosen microorganism and inoculate this into $100 \mathrm{ml}$ bottles of TSB broth (one bottle per container) and incubate for $18-24$ hours at $30-35{ }^{\circ} \mathrm{C}$. Use this as the inoculum in the integrity test.

Prepare a sterile container, with lid, of suitable size to contain the syringes under test and which is also capable of being placed in an incubator. In a laminar flow cabinet, spray and wipe the outer surface of the syringes under test with sterile $70 \%$ industrial methylated spirit (IMS) and allow to dry. Place syringes in the container(s) and cover with single strength TSB, ensuring that the syringes remain submerged.

If using Brevundimonas diminuta, inoculate the surrounding broth with a volume of the $18-24$ hour culture. $(1 \mathrm{ml}$ of the culture per $100 \mathrm{ml}$ of single strength TSB.) Incubate the containers for 14 days at $30-35^{\circ} \mathrm{C}$. Following incubation, remove the syringes from the broth culture and examine each for turbidity (indicating Brevundimonas diminuta access into the syringe.)

If using E-Coli, inoculate the surrounding broth with $100 \mathrm{ml}$ of the single strength TSB containing the organism and leave for at least 30 minutes. Remove the syringes and rinse away the E-coli and broth. Dry and incubate the syringes for 14 days at 30-35 ${ }^{\circ} \mathrm{C}$. Check for turbidity, indicating growth.

With both challenge organisms, integrity of the syringe/closure system is confirmed providing that the broth in all syringes remains free from growth. If the syringes pass the test, i.e. show no growth, two should be inoculated with less than 100 cfu of the chosen test organism (positive control) and incubated for 3 days at $30-35{ }^{\circ} \mathrm{C}$. The test is satisfactory i.e. validated, if both syringes show signs of growth.
The physical dye intrusion test is a simple and rapid test to evaluate batch to batch syringe performance. This test will enable greater numbers of syringes to be tested if the batch size is large and evidence for the release of syringes for use will not rely on an incubation period.

Due to the potential for flexing or bending of the extended plunger after filling, syringes, should not be filled to their full extent to help minimise the potential for leakage during storage or transport. Generally, the syringe, when used as a storage container, should not be filled to more than $85 \%$ of its nominal capacity.

\section{BOX 7. Dynamic intrusion test (example of syringes)}

This dynamic dye-intrusion test involves filling each syringe (normally 20 syringes from a single manufacturer's batch) with water or a drug solution to $85 \%$ of the full scale marked on the syringe, and securely applying the appropriate hub. Then apply an internal vacuum by drawing back the plunger to the graduation representing $100 \%$ of syringe volume. Secure the barrel in place with a retaining pin or screw placed though a pre-drilled hole in the plunger to maintain the internal vacuum.

Prepare a single positive control syringe from the batch under test by incorporating a fine thread of stainless-steel wire (diameter $0.12 \mathrm{~mm}$ ) running parallel to the barrel between the plunger seal and the inner barrel wall. Immerse the test syringes, with the positive control in the centre of the group, in an upright position in a bath of dye, such as methylene blue or amaranth.

Place the dye bath onto a roller mixer and rotate at 45 revolutions/ minute for 2 hours. Release the internal vacuum by removal of the retaining devices (screw or wire) Thoroughly wash the external surfaces. Dispense a quantity of each syringe and the positive control into a suitable cuvette or matched Nessler cylinder. Examine visually for the presence of dye using the contents from an untested control syringe as the reference.

Syringes comply with the test if contents of all units show no evidence of dye ingress. The test is valid only if the positive control syringe contents are coloured with the dye.

The most complete assessment of the syringe and blind hub (cap) as a container and closure is evaluated by application of both a microbiological and a physical integrity test when a new or altered syringe and hub combination is considered. A physical integrity test is acceptable for subsequent and routine approval of batches of syringes and caps of the same combination.

Another method that can be used, for example for bags and plastic overwrapping, is "ASTM F19299: standard test method for detecting seal leaks in porous medical packaging by dye penetration". The method allows the visual and microscopic detection of leaks of the seal using a mixture of toluidine blue and Triton X-100 surfactant. 


\section{Optional additional test - Microbial in-use viability testing}

The probability of microbial growth in hospital pharmacy aseptic preparations is related to the growth supporting nature of the preparation. Each drug formulation possesses a different potential for supporting or inhibiting growth. Viability of microbes depends on the nutrient content, $\mathrm{pH}$, redox potential and water activity of the preparation. Pure lipid emulsion and lipid-containing preparations support microbial growth. ${ }^{6}$ Many aqueous pharmaceutical preparations do not have nutritive properties and do not promote microbial growth. ${ }^{7}$ However, microbes may remain viable in most pharmaceutical preparations including cytotoxic or even antibiotic solutions. Even when physico-chemical characteristics of preparations are unlikely to support microbial growth (e.g. preparations with strong alkaline $\mathrm{pH}$ like 5-Fluorouracil, ganciclovir-sodium, foscarnet-sodium which have microbiocidal properties), viability of microorganisms cannot be totally excluded. No direct link between chemical structures or pharmacological principles and antimicrobial activity was observed during different studies with small

6 Sarakbi I, Federici, M, Krämer, I. Viability of microorganisms in novel chemical and biopharmaceutical anticancer drug solutions. Eur J Parenter Pharm Sci 2015;20: 5-12.

7 Sarakbi I, Heeb R, Thiesen J, Krämer I. Viability of selected microorganisms in non-cytotoxic aseptic preparations. Pharm Technol Hosp Pharm 2016;1: 9-20.

Note : Manufacturers of licensed products proposing in-use shelflife and storage conditions following reconstitution must submit results of microbiological challenge testing as part of their Marketing Authorisation application. The International Conference of Harmonization guidance references this testing in ICH Q8 Pharmaceutical Development, Microbial Attributes, Section 2.5 (2). It states: "Where relevant, microbial challenge testing under testing conditions that, as far as possible, simulate patient use should be performed during development and documented...”. The Center for Drug Evaluation and Research (CDER), USA expects that new drug applications are accompanied by information about its potential to support microbial growth. Microbial in-use studies should be performed to determine if the product will support microbial growth and/or proliferate in the event of inadvertent microbial contamination during the holding period prior to patient administration. The final product should be inoculated with small numbers of challenge microorganisms. CDER recommends using the five challenge microorganisms listed in USP $<51>$ Antimicrobial Effectiveness Testing (testing multi-use drug products that contain antimicrobial preservatives) for microbial in-use testing. These are Candida albicans, Aspergillus brasiliensis, E. coli, Pseudomonas aeruginosa, Staphylococcus aureus. The same challenge microorganisms are specified in the European Pharmacopoeia monograph Ph. Eur. 5.1.3 Efficacy of Antimicrobial Preservation. molecule active substances. Moreover, no correlation exists between antifungal and antibacterial activity, and antimicrobial activity is species specific. It is of note that the protein preparations tested exhibited neither antibacterial nor antifungal activity and did not facilitate microorganism reproduction. The selected micro-organisms were not able to use antibody-containing preparations as a nutrient source. The potential of antibody preparations to support antimicrobial growth is similar to that of preparations containing low molecular weight chemical substances.

Parenteral preparations contain a wide range of active substances, additives and diluent(s), as well as proposed holding times and conditions. ${ }^{8}$ Microbial growth studies are performed to get an understanding of the growth rate of selected microorganisms in readyto-administer preparations. Knowledge about the viability (i. e. ability to survive) of microbes in parenteral preparations can give additional useful information for the assignment of shelf lives. Therefore, the expert panel suggests consideration of literature data on the viability of microorganisms in comparable hospital pharmacy aseptic preparations or the performance of novel standalone viability studies. Studies should be designed to determine the extent to which microorganisms survive in the ready-to-administer preparation (Box 8). The final preparation is inoculated with a low number of test microorganisms, for example $10^{2}-10^{5}$ colony forming units (CFU) per $\mathrm{mL}$, and aliquots are taken at suitable intervals and storage conditions to determine the microbial count.

Growth curves of selected test microorganisms in the preparation are generated. In order to increase the significance of the test results (see Note), preparations should be inoculated with microorganisms which are commonly associated with nosocomial infections and represent potential contaminants (e.g. Staphylococcus aureus, Pseudomonas aeruginosa, Enterococcus faecium, E. coli, Candida albicans). The test conditions should simulate the worst possible conditions for patients (lowest pharmaceutical substance concentrations used in clinical practice) and optimal circumstances for the growth of micro-organisms (e.g. storage at room temperature).

8 Speed Ricci M, Frazier M, Moore J et al. In-use physicochemical and microbiological stability of biological parenteral products. Am J Health-System Pharm. 2015;72:396-407. 
Box 8. Microbial viability testing of ready-to-administer aseptic preparations

Parenteral products selected for testing are aseptically prepared in pharmacy according to the manufacturers' recommendations. Samples are in most cases prepared by injecting the calculated amount of each concentrated solution into a polyolefin bag containing $0.9 \% \mathrm{NaCl}$ or $5 \%$ dextrose infusion solutions.

The resulting concentrations should be on the lower limit of the concentration recommended in the Summary of Product Characteristics (SmPC).

The challenge microorganisms (representing e.g. Gram-positive cocci, Gram-negative bacilli, yeast, and mould) and the inocula are prepared according to the Ph.Eur. 5.1.3.

Each microorganism is injected individually into the test preparation.

The inoculated preparations are stored at room temperature $\left(20-25^{\circ} \mathrm{C}\right)$ and protected from light.

$1 \mathrm{~mL}$ samples are taken immediately and at predetermined intervals (e. g. 1, 3, 5, 24, 48, and 144 hours after inoculation). Samples are diluted 1:10 consecutively three times by using tubes prefilled with $0.9 \% \mathrm{NaCl}$ solution.

Finally, $0.1 \mathrm{~mL}$ aliquots of the maximum diluted samples are transferred to tryptic soy agar plates.

The plates are incubated for 24 hours at $37{ }^{\circ} \mathrm{C}$ and the colony forming units (CFU) counted.

\section{Routine tests for aseptically prepared products}

For each batch prepared for stock, the following tests must be performed

\section{Sterility tests}

The filtration method is the reference method according $\mathrm{Ph}$. Eur. 5.1.1. and all other methods have to be validated.

One alternative rapid microbiological method widely used in hospitals is sterility testing by aliquot sampling and transfer to blood culture bottles (aerobic and anaerobic blood culture bottles in parallel). The method allows detection of $\mathrm{CO} 2$ using colorimetric methods or by change in pressure in a culture medium vial equipped with a $\mathrm{CO2}$ sensor of sensitivity adapted to the quantity produced by the micro-organisms. Microbial growth usually becomes evident after 24-48 hours of incubation. Total incubation time is 14 days, to increase the chance of detection of slowgrowing organisms. The statistical limitations of this type of testing must, however, be acknowledged.

\section{Retrospective or prospective sterility testing?}

When preparations are prepared in batches for stock and an extended shelf life is assigned, the question arises of whether sterility tests are required prior to the release of the preparations (prospective sterility testing). Official regulations are not implemented at this time. The approach utilised in the UK may, however, serve as a model. For sterile preparations, including those made aseptically, with a shelf life of less than 90 days, it is accepted by the UK Regulatory Authority (the MHRA) that the results from sterility tests or end of session media fills may not need to be available or considered as part of the preparation release criteria. (90 days was selected as a practical timescale for remaining preparation shelf life based on a 14-day incubation period.) The rationale for this must be justified, however, and there is an expectation that retrospective end of session media fills and sterility tests will form part of the body of evidence for sterility assurance.

The expectation for preparations with a shelf life of 90 days or more is that a prospective acceptable sterility test or end of session media fill should be completed prior to preparation release. Under these circumstances the sterility test or end of session media fill must relate to the batch in question i. e. a sample of the batch must be part of the sterility test, or the end of session media fill conducted must include the processing of this specific batch. ${ }^{9}$

\section{Other optional end product tests - Endotoxins}

An endotoxin test, in accordance with Eur. Ph. 2.6.14, should be performed if the aseptic process is at risk of endotoxin contamination especially when using a raw (non-sterile) material as a starting material.

\section{Assignment of microbiological shelf-life}

Shelf lives should be assigned according to the results of sterility/integrity tests during the validation phase (See Box 9).

9 MHRA Q\&As Guidance for 'Specials' Manufacturers 2015 https://assets.publishing.service.gov.uk/government/uploads/sys tem/uploads/attachment_data/file/400232/Guidance_for_spe cials_manufacturers.pdf 


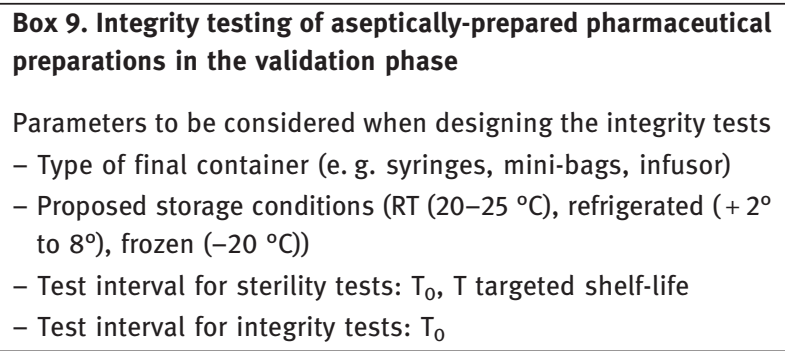

Parameters to be considered when designing the integrity tests

- Type of final container (e. g. syringes, mini-bags, infusor)

- Proposed storage conditions (RT $\left(20-25^{\circ} \mathrm{C}\right)$, refrigerated $\left(+2^{\circ}\right.$ to $\left.8^{\circ}\right)$, frozen $\left(-20^{\circ} \mathrm{C}\right)$ )

- Test interval for sterility tests: $T_{0}$, $T$ targeted shelf-life

- Test interval for integrity tests: $T_{0}$

Depending on the type of batch prepared, two different strategies may be followed.

a) Assignment of shelf-lives for aseptic preparations of batches derived from the same initial bulk admixture and prepared for stock

A summary of the assignment procedure strategy in case of batch preparation from the same initial bulk admixture is given in Figure 1.

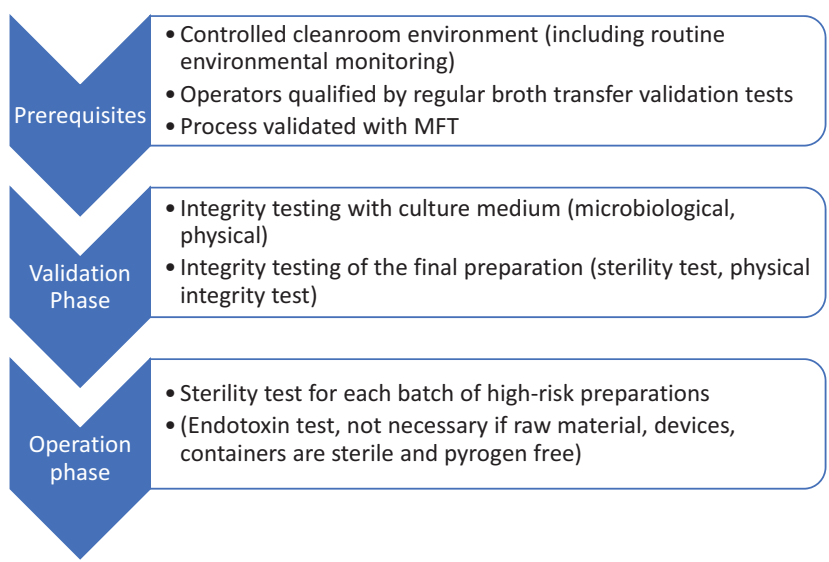

Figure 1: Summary of the strategy in case of batch production from the same initial bulk admixture for stock.

b) Assignment of shelf-lives for aseptic preparations of batches prepared by the same process and with empirically assessed microbiological stability

A summary of the assignment procedure strategy in case of batches prepared by the same process is given in Figure 2

There is no limitation on the shelf-life that can be allocated to a preparation when this based on robust microbiological and physicochemical stability data and regulatory requirements allow. However strategies for the empirical allocation of shelf-lives are in a few national

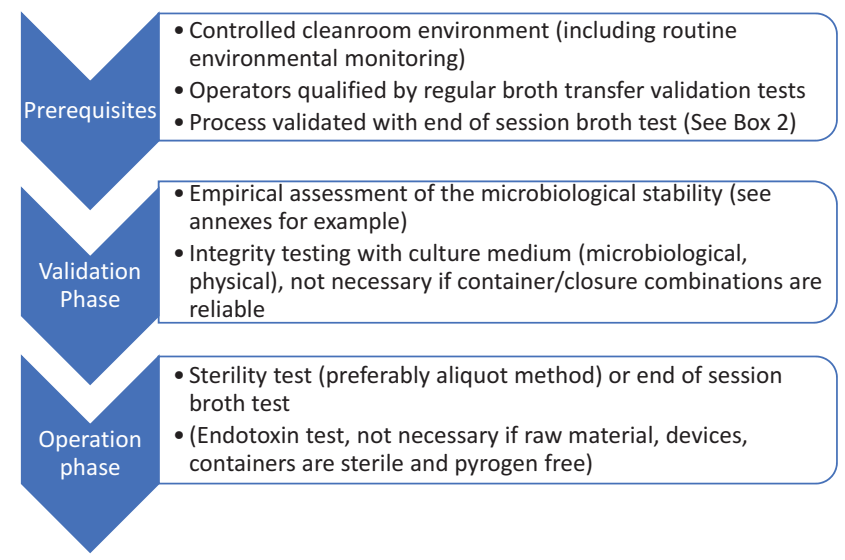

Figure 2: Summary of the strategy in case of batch preparation using the same process for immediate use or for stock.

regulations in the UK, ${ }^{10}$ in the Netherlands (see Annex 1) and in the USA in the USP (see Annex 2). None of these empirical shelf-lives is validated and none may be considered as a universal standard.

They are not, therefore endorsed by the members of the expert group.

Empirical allocation of shelf-life, if essential to be carried out, should be based on assessment of risk factors, as outlined in Box 1.

Finally, microbiological and physicochemical stability are to be considered concurrently when determining the shelf life of an individual preparation. In order to minimise the risk for the patient, in each case shelf life should be limited according to the shorter period of proven stability, either derived from the microbiological or physicochemical stability data.

\section{Annex 1: Empirical allocation of shelf-lives from Netherlands ${ }^{11}$}

For batches prepared by using the same process in a controlled cleanroom environment, an empirical shelf life determination is accepted.

10 Quality Assurance of Aseptic Preparation Services Edition 5 (2016), Editor Beaney AM on behalf of the Royal Pharmaceutical Society and NHS QA Committee.

11 Dutch GMP-Hospital Pharmacy chapter H3. 


\section{Low risk preparations}

The recommended shelf life is 7 days for preparations stored at room temperature and 1 month for preparations stored under refrigerated conditions $\left(2-8{ }^{\circ} \mathrm{C}\right)$

There is no recommendation for storage in a freezer $\left(-20^{\circ} \mathrm{C}\right)$

\section{High risk preparations}

The recommended shelf life is 7 days for preparations stored under refrigerated conditions $\left(2-8{ }^{\circ} \mathrm{C}\right)$

There is no recommendation for storage at room temperature or for storage in a freezer $\left(-20^{\circ} \mathrm{C}\right)$

\section{Annex 2 Empirical allocation of shelf-lives from USP $\langle 797\rangle^{12}$}

Low risk preparation (Note for USP "Low risk" preparation is Aseptic manipulations within an ISO Class 5 environment using three or fewer sterile products and entries into any container)
$48 \mathrm{~h}$ at Room Temperature

14 days at $+2-+8{ }^{\circ} \mathrm{C}$

45 days Freezing -10 to $-25^{\circ} \mathrm{C}$

Medium Risk Preparation (Note for USP "Medium risk" preparation is Aseptic manipulations within an ISO Class 5 environment using prolonged and complex mixing and transfer, more than three sterile products and entries into any container, and pooling ingredients from multiple sterile products to prepare multiple CSPs.)

$30 \mathrm{~h}$ at $\mathrm{RT}$

9 days at $+2-+8{ }^{\circ} \mathrm{C}$

45 days Freezing -10 to $-25^{\circ} \mathrm{C}$

High risk preparation (Note for USP "High risk" preparation is Confirmed presence of nonsterile ingredients and devices, or confirmed or suspected exposure of sterile ingredients for more than one hour to air quality inferior to ISO Class 5 before final sterilization.)

$24 \mathrm{~h}$ at $\mathrm{RT}$

3 days at $+2-+8{ }^{\circ} \mathrm{C}$

45 days Freezing -10 to $-25^{\circ} \mathrm{C}$ 